\title{
CONTINUATION UNIQUE À PARTIR DE L'INFINI CONFORME POUR LES MÉTRIQUES D'EINSTEIN
}

\author{
OLIVIER BIQUARD
}

\begin{abstract}
We prove the unique continuation property at the conformal infinity for
\end{abstract} asymptotically hyperbolic Einstein metrics.

Le but de cette note est de prouver un résultat d'unicité pour les métriques d'Einstein asymptotiquement hyperboliques dont le comportement à l'infini est fixé à un ordre suffisamment grand. Cette question, ainsi que sa variante infinitésimale, semble posée depuis quelque temps, et est importante dans le cadre du programme d'Anderson [1] visant à donner des critères généraux d'existence de telles métriques à bord conforme donné.

Soit une variété $M^{n+1}$ à bord $X^{n}=\partial M$, une métrique $g$ dans l'intérieur de $M$ est dite asymptotiquement hyperbolique s'il existe une équation $\{x=0\}$ de $X \subset M$, et une métrique $\gamma$ sur $X$, telles que, pour $x \rightarrow 0$,

$$
g \sim \frac{d x^{2}+\gamma}{x^{2}}
$$

Seule la classe conforme $[\gamma]$ de $\gamma$ est alors bien définie par $g$ et est appelée l'infini conforme de $g$. Un exemple de telle métrique est évidemment la métrique hyperbolique $\frac{d x^{2}+d x_{1}^{2}+\cdots+d x_{n}^{2}}{x^{2}}$. Plus généralement, à partir d'un bord conforme $(X,[\gamma])$, Fefferman et Graham [5] ont trouvé un développement formel pour une métrique d'Einstein asymptotiquement hyperbolique $g$ sur un voisinage tubulaire $M=] 0, \epsilon[\times X$. En coordonnées géodésiques, la métrique s'écrit $g=\frac{d x^{2}+g_{x}}{x^{2}}$, où $g_{x}$ est une métrique sur $\{x\} \times X$, admettant un développement

$$
g_{x}=\left\{\begin{array}{l}
\gamma+x^{2} g_{2}+x^{3} g_{3}+\cdots+x^{n} g_{n}+\cdots, \quad n \text { impair } \\
\gamma+x^{2} g_{2}+x^{3} g_{3}+\cdots+x^{n} \ln x G+x^{n} g_{n}+\cdots, \quad n \text { pair }
\end{array}\right.
$$

où tous les termes du développement sont formellement déterminés, à l'exception du terme $g_{n}$. L'existence générale d'un développement polyhomogène est prouvée dans $[4,7]$. Il en résulte que deux métriques ayant même infini conforme et même terme indéterminé, écrites dans des coordonnées géodésiques, coïncident à un ordre infini près de $X$. Nous prouvons qu'elles sont égales :

Théorème 1. Soient deux métriques d'Einstein asymptotiquement hyperboliques avec le même infini conforme et le même terme indéterminé; alors, à un difféomorphisme égal à l'identité au bord près, les deux métriques sont égales dans un voisinage du bord.

Received by the editors September 24, 2007.

L'auteur est soutenu par le contrat 06-BLAN60154-01 de l'ANR. 
Bien entendu, il y a un analogue infinitésimal. Si on a une métrique d'Einstein asymptotiquement hyperbolique $g$, alors une déformation infinitésimale d'Einstein est un 2-tenseur symétrique $\dot{g}$, satisfaisant la linéarisation de l'équation $\operatorname{Ric}(g)=-n g$, à savoir

$$
\frac{1}{2}\left(\nabla^{*} \nabla \dot{g}+\operatorname{Ric} \circ \dot{g}+\dot{g} \circ \operatorname{Ric}-2 \stackrel{\circ}{R} \dot{g}\right)-\delta^{*} \delta \dot{g}-\frac{1}{2} \delta^{*} d \operatorname{Tr} \dot{g}=-n \dot{g} .
$$

Écrivons $g$ en jauge géodésique, comme ci-avant. En agissant par un difféomorphisme infinitésimal, on peut mettre aussi $\dot{g}$ dans une jauge géodésique $\left.\left(\partial_{x}\right\lrcorner \dot{g}=0\right)$; dans une telle jauge, $\dot{g}$ admet un développement similaire à (2). En particulier, si la déformation infinitésimale $\dot{g}$ ne modifie pas la métrique conforme au bord, alors, toujours en jauge géodésique, on a

$$
\dot{g}=\frac{1}{x^{2}}\left(x^{n} \dot{g}_{n}+\cdots\right),
$$

où tout le développement est déterminé par le seul terme indéterminé $\dot{g}_{n}$, un 2-tenseur symétrique sur le bord (il est de plus à divergence et trace nulles). On peut alors énoncer :

Théorème 2. Soit g une métrique d'Einstein asymptotiquement hyperbolique, et $\dot{g}$ une déformation infinitésimale d'Einstein de $g$ définie près du bord; écrivons $\dot{g}$ en jauge géodésique : si $|\dot{g}|_{g}=o\left(x^{n}\right)$ alors $\dot{g}=0$ (toujours dans la jauge géodésique).

Une version locale est vraie :

Théorème 3. Les théorèmes 1 et 2 restent vrais, mutatis mutandis, pour des métriques définies seulement localement près d'un point du bord.

Un corollaire de la démonstration, suggéré par M. Herzlich, est le cas plus facile, mais apparemment ouvert, d'un bord à distance finie :

Théorème 4. Deux métriques d'Einstein sur une variété à bord $M$, de même constante d'Einstein, dont la restriction au bord $X=\partial M$ et la seconde forme fondamentale sur $X$ coïncident, diffèrent près du bord par un difféomorphisme induisant l'identité sur $X$.

Bien sûr, la version infinitésimale du théorème 4, que l'on n'énoncera pas, reste valable aussi pour un bord à distance finie. En revanche, une version locale de ce théorème semble plus difficile à obtenir par les techniques développées dans cet article.

La difficulté habituellement rencontrée avec les équations d'Einstein est le manque d'ellipticité de l'équation sur la métrique, écrite dans des coordonnées géodésiques. On y remédie en observant que, en coordonnées géodésiques, la seconde forme fondamentale des tranches satisfait une équation elliptique non linéaire, dépendant de la métrique, avec second membre impliquant la courbure moyenne et ses dérivées. Il y a alors une compétition entre les estimations de Carleman adaptées au cas asymptotiquement hyperbolique [8], et les termes d'erreur provenant de la métrique et de la courbure moyenne, mais le contrôle de ceux-ci par la seconde forme fondamentale, grâce à la partie radiale de l'équation d'Einstein, s'avère suffisant.

Dans cet article, on se limite au cas où la métrique au bord est de classe $C^{\infty}$. Les estimations de Carleman n'utilisent que deux dérivées de la métrique, donc il est clair que nos résultats d'unique continuation restent valables pour des métriques 
au bord de régularité finie (par exemple $C^{3, \alpha}$ ), sous réserve de supposer a priori que les deux métriques coïncident à un ordre infini près du bord. En réalité, la question naturelle consiste à supposer seulement une coïncidence à l'ordre $o\left(x^{n}\right)$, et une régularité $C^{2, \alpha}$, voire même $C^{\alpha}$, sur le bord. La question d'unique continuation pose alors des problèmes plus techniques, qui seront abordés ailleurs. Il reste aussi à explorer la généralisation de ces résultats à d'autres comportements asymptotiques, comme ceux étudiés dans [3].

\section{Les équations}

Nous utiliserons la coordonnée $r=-\ln x$, de sorte que le modèle $\frac{d x^{2}+\gamma}{x^{2}}$ devient $d r^{2}+e^{2 r} \gamma$. Soit une métrique en coordonnées géodésiques, $g=d r^{2}+\bar{g}$. La seconde forme fondamentale des tranches $\{x\} \times X$ est $\mathbb{I}=-\frac{1}{2} \mathcal{L}_{\partial_{r}} \bar{g}$, dont on notera la trace $H=\operatorname{Tr}^{\bar{g}}(\mathbb{I})$. Pour une métrique asymptotiquement hyperbolique, on a $\mathbb{I} \rightarrow-1$ et $H \rightarrow-n$. L'équation $\operatorname{Ric}^{g}=-n g$ se décompose alors en le système, sur chaque tranche $\{x\} \times X$,

$$
\begin{aligned}
\overline{\operatorname{Ric}}-H \mathbb{I}+\mathcal{L}_{\partial_{r}} \mathbb{I}+2 \mathbb{I}^{2} & =-n \bar{g} \\
\bar{\delta} \mathbb{I}+d H & =0 \\
\partial_{r} H-|\mathbb{I}|^{2} & =-n .
\end{aligned}
$$

Ici $\overline{\operatorname{Ric}}, \bar{\delta}, \ldots$ se rapportent à la métrique $\bar{g}$, et $\mathbb{I}^{2}$, vu comme endomorphisme symétrique pour $\bar{g}$, est le carré de $\mathbb{I}$. Par la formule (3) pour la linéarisation du tenseur de Ricci, on a

$$
\begin{aligned}
\mathcal{L}_{\partial_{r}} \overline{\operatorname{Ric}} & =\mathrm{d}_{\bar{g}} \overline{\operatorname{Ric}}\left(\mathcal{L}_{\partial_{r}} \bar{g}\right)=-2 \mathrm{~d}_{\bar{g}} \overline{\operatorname{Ric}}(\mathbb{I}) \\
& =-2\left\{\frac{1}{2}\left(\bar{\nabla}^{*} \bar{\nabla} \mathbb{I}+\overline{\operatorname{Ric}} \circ \mathbb{I}+\mathbb{I} \circ \overline{\operatorname{Ric}}-2 \stackrel{\circ}{\bar{R}} \mathbb{I}\right)-\bar{\delta}^{*} \bar{\delta} \mathbb{I}-\frac{1}{2} \bar{\delta}^{*} d \operatorname{Tr}^{\bar{g}}(\mathbb{I})\right\} .
\end{aligned}
$$

En appliquant $\mathcal{L}_{\partial_{r}}$ à l'équation (5), et en utilisant (6) qui s'apparente à une condition de jauge sur $\mathbb{I}$, on déduit de (8) l'équation

$$
\begin{aligned}
-\bar{\nabla}^{*} \bar{\nabla} \mathbb{I}+\mathcal{L}_{\partial_{r}}^{2} \mathbb{I}-H \mathcal{L}_{\partial_{r}} \mathbb{I}-\left(\partial_{r} H\right) \mathbb{I}+2\left(\mathbb{I} \circ \mathcal{L}_{\partial_{r}} \mathbb{I}+2 \mathbb{I} \circ \mathbb{I} \circ \mathbb{I}+\mathcal{L}_{\partial_{r}} \mathbb{I} \circ \mathbb{I}\right) \\
-\overline{\operatorname{Ric}} \circ \mathbb{I}-\mathbb{I} \circ \overline{\operatorname{Ric}}+2 \overline{\bar{R}} \mathbb{I}-2 n \mathbb{I}-\bar{\delta}^{*} d H=0 .
\end{aligned}
$$

On remarquera qu'en faisant disparaître $H$ de cette équation grâce à l'équation (6), on trouverait une autre équation, non elliptique, sur II. C'est pourquoi on préfère ici traiter les termes en $H$ comme un second membre.

\section{Estimation de $g$ et $H$}

Pour une fonction $f$ sur $\left[r_{0},+\infty\left[\right.\right.$, telle que $|f|=O\left(e^{-\lambda r}\right)$ pour tout $\lambda>0$, une intégration par parties donne immédiatement

$$
\int_{r_{0}}^{+\infty}\left|\partial_{r} f\right|^{2} e^{2 \lambda r} d r \geqslant \lambda^{2} \int_{r_{0}}^{+\infty}|f|^{2} e^{2 \lambda r} d r
$$

Aucune condition sur $f$ en $r_{0}$ n'est nécessaire.

Supposons maintenant que deux métriques d'Einstein asymptotiquement hyperboliques $g$ et $g_{0}$ aient mêmes infini conforme et terme indéterminé. Une métrique, 
choisie dans la classe conforme, détermine des coordonnées géodésiques dans un voisinage du bord [6], dans lesquelles $g$ et $g_{0}$ coïncident donc à un ordre infini au bord. Nous pouvons nous restreindre à un voisinage $] r_{0},+\infty\left[\times X\right.$ du bord sur lequel $g$ et $g_{0}$ sont mutuellement bornées, et toutes les dérivées de $g-g_{0}$ restent bornées par rapport à $g_{0}$. De l'estimation (10), appliquée dans des systèmes de coordonnées, résulte alors immédiatement, pour $\lambda>2$,

$$
\int_{r_{0}}^{+\infty}\left|\mathbb{I}-\mathbb{I}_{0}\right|_{g_{0}}^{2} e^{2 \lambda r} d r \geqslant C^{-1} \lambda^{2} \int_{r_{0}}^{+\infty}\left|g-g_{0}\right|_{g_{0}}^{2} e^{2 \lambda r} d r
$$

pour une constante $C$ indépendante de $\lambda$. Dans la suite, la constante $C$ pourra grandir à chaque ligne, mais restera indépendante de $\lambda$. On peut aussi dériver l'équation $\mathbb{I}-\mathbb{I}_{0}=-\frac{1}{2} \mathcal{L}_{\partial_{r}}\left(g-g_{0}\right):$ comme toutes les courbures et secondes formes fondamentales sont bornées, les commutations n'introduisent pas de nouveau terme et on obtient

$$
\int_{r_{0}}^{+\infty} \sum_{0}^{k}\left|\nabla_{0}^{j}\left(\mathbb{I}-\mathbb{I}_{0}\right)\right|_{g_{0}}^{2} e^{2 \lambda r} d r \geqslant C^{-1} \lambda^{2} \int_{r_{0}}^{+\infty} \sum_{0}^{k}\left|\nabla_{0}^{j}\left(g-g_{0}\right)\right|_{g_{0}}^{2} e^{2 \lambda r} d r .
$$

De même, de l'équation (7) on déduit $\partial_{r}\left(H-H_{0}\right)=|\mathbb{I}|_{g}^{2}-\left|\mathbb{I}_{0}\right|_{g_{0}}^{2}$; puisque $g$ et toutes ses dérivées demeurent uniformément bornées par rapport à $g_{0}$ dans le voisinage considéré, on a $\left.|| \mathbb{I}\right|_{g} ^{2}-\left|\mathbb{I}_{0}\right|_{g_{0}}^{2} \mid \leqslant C\left(\left|\mathbb{I}-\mathbb{I}_{0}\right|_{g_{0}}+\left|g-g_{0}\right|_{g_{0}}\right)$, et de (10) et (11) résulte l'estimation

$$
\int_{r_{0}}^{+\infty}\left|\mathbb{I}-\mathbb{I}_{0}\right|_{g_{0}}^{2} e^{2 \lambda r} d r \geqslant C^{-1} \lambda^{2} \int_{r_{0}}^{+\infty}\left|H-H_{0}\right|^{2} e^{2 \lambda r} d r
$$

En prenant des dérivées de (7), comme ci-dessus, la même estimation reste valable pour les dérivées :

$$
\int_{r_{0}}^{+\infty} \sum_{0}^{k}\left|\nabla_{0}^{j}\left(\mathbb{I}-\mathbb{I}_{0}\right)\right|_{g_{0}}^{2} e^{2 \lambda r} d r \geqslant C^{-1} \lambda^{2} \int_{r_{0}}^{+\infty} \sum_{0}^{k}\left|\nabla_{0}^{j}\left(H-H_{0}\right)\right|^{2} e^{2 \lambda r} d r .
$$

\section{Estimation de Carleman et preuve des théorèmes 1 et 2}

Rappelons ici la version de Mazzeo de l'estimation de Carleman [8, théorème 7] : si $P$ est un opérateur elliptique pour une métrique asymptotiquement hyperbolique $g$, de la forme

$$
P s=\nabla^{*} \nabla s+\text { termes d'ordre inférieur bornés en } \nabla s \text { et } s,
$$

alors il existe $r_{0}>0$ tel que pour tous $\lambda \gg 0$ et $s$ à support dans $\left\{r>r_{0}\right\}$ on ait

$$
\int_{r \geqslant r_{0}}|P s|^{2} e^{2 \lambda r} \mathrm{vol}^{g} \geqslant C^{-1} \int_{r \geqslant r_{0}}\left(\lambda^{-1}\left|\nabla^{2} s\right|^{2}+\lambda|\nabla s|^{2}+\lambda^{3}|s|^{2}\right) e^{2 \lambda r} \mathrm{vol}^{g} .
$$

En fait le terme $\left|\nabla^{2} s\right|^{2}$ n'est pas écrit dans l'énoncé cité, mais il est évident de revoir la démonstration pour montrer sa présence avec le coefficient indiqué. En outre, les constantes $r_{0}$ et $C$ peuvent être choisies uniformes si $g$ et les coefficients de $P$ varient de manière bornée.

Appliquons à présent cette estimation à la démonstration du théorème 1. Comme précédemment, nous avons donc deux métriques d'Einstein $g$ et $g_{0}$ qui coïncident à un ordre infini dans des coordonnées géodésiques, et nous analysons la différence des 
équations (9) pour les métriques $g$ et $g_{0}$. Par exemple, le premier terme $\bar{\nabla}^{*} \bar{\nabla} \mathbb{I}-\bar{\nabla}_{0}^{*} \bar{\nabla}_{0} \mathbb{I}_{0}$ se décompose en

$$
\bar{\nabla}_{0}^{*} \bar{\nabla}_{0}\left(\mathbb{I}-\mathbb{I}_{0}\right)+\left(\bar{\nabla}^{*} \bar{\nabla}-\bar{\nabla}_{0}^{*} \bar{\nabla}_{0}\right) \mathbb{I} ;
$$

dans cette expression, le premier terme est la linéarisation en $g_{0}$ et, puisque toutes les dérivées de $g$, et donc de $\mathbb{I}$ aussi, sont supposées bornées, le second terme est contrôlé en chaque point par $C\left(\left|g-g_{0}\right|+\left|\bar{\nabla}_{0}\left(g-g_{0}\right)\right|\right)$.

Plus généralement, notons $\Phi(g, H)(\mathbb{I})$ le membre de gauche de l'équation (9), et $P=\frac{\partial \Phi}{\partial \mathbb{I}}\left(g_{0}, H_{0}\right)$ la linéarisation de l'équation en $\mathbb{I}_{0}$. Alors $\Phi\left(g_{0}, H_{0}\right)\left(\mathbb{I}_{0}\right)=0$ et

$$
\Phi(g, H)(\mathbb{I})=P\left(\mathbb{I}-\mathbb{I}_{0}\right)+Q(g, H)(\mathbb{I}),
$$

et une lecture attentive de chaque terme comme dans l'équation (17) donne l'estimation

$$
\begin{aligned}
&|Q(g, H)(\mathbb{I})| \leqslant \epsilon\left(\left|\mathbb{I}-\mathbb{I}_{0}\right|+\left|\nabla_{0}\left(\mathbb{I}-\mathbb{I}_{0}\right)\right|\right) \\
&+C\left(\left|g-g_{0}\right|+\right.\left|\nabla_{0}\left(g-g_{0}\right)\right|+\left|\nabla_{0}^{2}\left(g-g_{0}\right)\right| \\
&\left.+\left|H-H_{0}\right|+\left|\nabla_{0}\left(H-H_{0}\right)\right|+\left|\nabla_{0}^{2}\left(H-H_{0}\right)\right|\right)
\end{aligned}
$$

La constante $\epsilon$ peut être prise petite, mais ce fait ne sera pas utilisé dans la suite. Combinant avec les estimations (12) et (14), on déduit l'estimation

$$
\begin{aligned}
\int_{r \geqslant r_{0}} & |Q(g, h)(\mathbb{I})|^{2} e^{2 \lambda r} \text { vol } \\
& \leqslant \int_{r \geqslant r_{0}}\left(\epsilon\left(\left|\mathbb{I}-\mathbb{I}_{0}\right|^{2}+\left|\nabla_{0}\left(\mathbb{I}-\mathbb{I}_{0}\right)\right|^{2}\right)+C \lambda^{-2} \sum_{0}^{2}\left|\nabla_{0}^{j}\left(\mathbb{I}-\mathbb{I}_{0}\right)\right|^{2}\right) e^{2 \lambda r} \text { vol }
\end{aligned}
$$

D'un autre côté, l'opérateur $P$ est un opérateur du second ordre, dont les termes de plus haut degré sont $-\bar{\nabla}_{0}^{*} \bar{\nabla}_{0}+\mathcal{L}_{\partial_{r}}^{2}$, et les autres termes sont uniformément bornés, donc il satisfait l'estimation de Carleman (16), que nous pouvons appliquer après avoir coupé $\mathbb{I}-\mathbb{I}_{0}$ entre $r_{0}$ et $r_{0}+1$, d'où :

$$
\begin{aligned}
\int_{r \geqslant r_{0}} \mid & \left.P\left(\mathbb{I}-\mathbb{I}_{0}\right)\right|^{2} e^{2 \lambda r} \mathrm{vol}+\int_{r_{0} \leqslant r \leqslant r_{0}+1} \sum_{0}^{2}\left|\nabla_{0}^{j}\left(\mathbb{I}-\mathbb{I}_{0}\right)\right|^{2} e^{2 \lambda r} \text { vol } \\
& \geqslant C^{-1} \int_{r \geqslant r_{0}}\left(\lambda^{-1}\left|\nabla_{0}^{2}\left(\mathbb{I}-\mathbb{I}_{0}\right)\right|^{2}+\lambda\left|\nabla_{0}\left(\mathbb{I}-\mathbb{I}_{0}\right)\right|^{2}+\lambda^{3}\left|\mathbb{I}-\mathbb{I}_{0}\right|^{2}\right) e^{2 \lambda r} \text { vol. }
\end{aligned}
$$

L'équation $P\left(\mathbb{I}-\mathbb{I}_{0}\right)+Q(g, H)(\mathbb{I})=0$, avec les deux estimations précédentes, fournit finalement pour $\lambda \gg 0$,

$$
\begin{aligned}
\int_{r_{0}} \leqslant r \leqslant r_{0}+1 & \sum_{0}^{2}\left|\nabla_{0}^{j}\left(\mathbb{I}-\mathbb{I}_{0}\right)\right|^{2} e^{2 \lambda r} \text { vol } \\
& \geqslant C^{-1} \int_{r \geqslant r_{0}+1}\left(\lambda^{-1}\left|\nabla_{0}^{2}\left(\mathbb{I}-\mathbb{I}_{0}\right)\right|^{2}+\lambda\left|\nabla_{0}\left(\mathbb{I}-\mathbb{I}_{0}\right)\right|^{2}+\lambda^{3}\left|\mathbb{I}-\mathbb{I}_{0}\right|^{2}\right) e^{2 \lambda r} \text { vol. }
\end{aligned}
$$

En faisant $\lambda \rightarrow+\infty$, on voit qu'il faut $\mathbb{I}-\mathbb{I}_{0}=0$ sur $r \geqslant r_{0}+1$, donc $g=g_{0}$.

Le théorème 2 est la version infinitésimale du théorème 1 , sa démonstration est plus facile : on écrit la linéarisation du système (5), (6) et (7), que l'on traite de manière similaire. 


\section{Variante locale pour un bord à l'infini}

Pour démontrer le théorème 3 dans le cas asymptotiquement hyperbolique, on localise le problème au voisinage d'un point du bord en se rappelant du modèle

$$
g_{n+1}=d r^{2}+\cosh ^{2}(r) g_{n}
$$

pour l'espace hyperbolique réel. L'infini conforme quand $r \rightarrow+\infty$ est alors juste une boule hyperbolique.

Le résultat local de continuation unique pour un opérateur elliptique dans [8] est montré en utilisant des coordonnées obtenues par les géodésiques issues d'une hypersurface $x^{2}+x_{1}^{2}+\cdots+x_{n}^{2}=\epsilon$, ce qui donne bien un modèle du type indiqué avant. Cependant, nous ne pouvons pas procéder ainsi, puisque l'hypothèse de coïncidence des métriques sur le bord $x=0$ serait alors perdue. Il faut donc travailler à partir du bord.

Soit une métrique d'Einstein asymptotiquement hyperbolique $g$, d'infini conforme $[\gamma]$. Près d'un point $p \in X=\partial M$, on fixe une métrique $\gamma$ dans la classe conforme, et une petite boule $B \subset X$ pour $\gamma$, centrée en $p$. Le but ici est de trouver une métrique $\tilde{\gamma}$ dans la classe conforme $[\gamma]$ sur $B$, singulière sur $\partial B$, et une fonction positive $u$ sur un voisinage convenable de $B$ dans $M$, s'annulant sur $B$, de sorte que, de manière similaire au modèle (23), on ait $g=\frac{d u^{2}+g_{u}}{u^{2}}$, avec $g_{u}$ une famille de métriques sur $B$, dépendant du paramètre $u$, telle que :

$-g_{0}=\tilde{\gamma}$

- chaque métrique $g_{u}$ est asymptotiquement hyperbolique sur $B$, d'infini conforme fixe $\left.\gamma\right|_{\partial B}$.

Pour construire $\tilde{\gamma}$ et $u$, nous commençons par voir le modèle de manière légèrement différente, en écrivant la métrique hyperbolique $g_{n}$ de (23) dans le modèle du demiespace :

$$
g_{n+1}=\frac{d u^{2}+\frac{\left(1+u^{2}\right)^{2}}{4} \frac{d v^{2}+d x_{2}^{2}+\cdots+d x_{n}^{2}}{v^{2}}}{u^{2}} .
$$

On revient à la forme plus classique $g_{n+1}=\frac{d x^{2}+d y^{2}+d x_{2}^{2}+\cdots+d x_{n}^{2}}{x^{2}}$ par le changement de variables $\left(x=\frac{2 u}{1+u^{2}} v, y=\frac{1-u^{2}}{1+u^{2}} v\right)$. Dans la formule (24), la métrique induite sur le bord $\{u=0\}$ est la métrique hyperbolique $\frac{d y^{2}+d x_{2}^{2}+\cdots+d x_{n}^{2}}{y^{2}}$ sur le demi-espace $\{y>0\}$.

Revenons à notre métrique asymptotiquement hyperbolique $g$ sur $M$, d'infini conforme $[\gamma]$, et à la petite boule $B \subset X=\partial M$, pour imiter le modèle qui précède. Soit $y$ la distance au bord $\partial B$ pour $\gamma$, prolongée en une fonction lisse et strictement positive à l'intérieur de $B$. Près de $X \subset M$ on a donc $g=\frac{d x^{2}+\gamma_{x}}{x^{2}}$, et dans $X=\{x=0\}$, près de $\partial B \subset X$, on a de plus la décomposition $\left.\gamma_{0}\right|_{X}=d y^{2}+\bar{\gamma}_{y}$, où $\bar{\gamma}_{y}$ est une famille de métriques sur $\partial B$, paramétrée par $y$. On obtient donc

$$
\begin{aligned}
\gamma_{x} & =\gamma_{0}+\gamma_{x}^{\prime}, \quad \gamma_{x}^{\prime}=O(x), \\
& =d y^{2}+\bar{\gamma}_{y}+\gamma_{x}^{\prime} \quad \text { sur un petit voisinage de } \partial B \text { dans } X,
\end{aligned}
$$

où $\gamma_{x}^{\prime}$ est une famille de métriques sur un voisinage de $\bar{B}$ dans $X$ (on peut en réalité supposer $\gamma_{x}^{\prime}=O\left(x^{2}\right)$ car le premier terme du développement de Fefferman-Graham s'annule, mais nous n'aurons pas besoin de ce contrôle plus précis). 
Effectuons, dans un voisinage de $\partial B$, le changement de variables qui amène à une forme de type (24),

$$
x=\frac{2 u_{0}}{1+u_{0}^{2}} v, \quad y=\frac{1-u_{0}^{2}}{1+u_{0}^{2}} v .
$$

Alors, toujours dans un voisinage de $\partial B$,

$$
g=\frac{d x^{2}+d y^{2}+\bar{\gamma}+\gamma^{\prime}}{x^{2}}=\frac{1}{u_{0}^{2}}\left(d u_{0}^{2}+\frac{\left(1+u_{0}^{2}\right)^{2}}{4} \frac{d v^{2}+\bar{\gamma}+\gamma^{\prime}}{v^{2}}\right) .
$$

Ce modèle nous incite à choisir dans la classe conforme de $\gamma$ sur $B$ la métrique asymptotiquement hyperbolique

$$
\tilde{\gamma}=\frac{\gamma}{4 y^{2}}
$$

puis à chercher la fonction $u$, perturbation de $u_{0}$, donnant les coordonnées géodésiques correspondantes :

$$
g=\frac{d u^{2}+\bar{g}_{u}}{u^{2}} .
$$

La fonction $u=u_{0} e^{\phi}$ doit être une « équation spéciale » de $X$, c'est-à-dire satisfaire :

$$
|d u|_{u^{2} g}=1,\left.\quad u^{2} g\right|_{B}=\tilde{\gamma}
$$

Graham [6] montre que cette équation aux dérivées partielles du premier ordre, non caractéristique le long de $X$, a toujours une solution dans un voisinage de $X$, mais ici la métrique $\tilde{\gamma}$ est singulière sur $\partial B$ et il faut donc préciser ce qui se passe au voisinage de $\partial B$. L'équation (30) se récrit

$$
\left(\nabla^{u_{0}^{2} g} u_{0}\right) \cdot \phi+\frac{u_{0}}{2}|d \phi|_{u_{0}^{2} g}^{2}=\frac{1-\left|d u_{0}\right|_{u_{0}^{2} g}^{2}}{2 u_{0}},\left.\quad \phi\right|_{X}=0 .
$$

D'après (27), on a

$$
u_{0}^{2} g=d u_{0}^{2}+\frac{\left(1+u_{0}^{2}\right)^{2}}{4} \frac{d v^{2}+\bar{\gamma}}{v^{2}}+\frac{\left(1+u_{0}^{2}\right)^{2}}{4 u_{0}^{2} v^{2}} \gamma^{\prime}
$$

avec (en convenant que $x_{1}=y$ ) :

$$
\begin{aligned}
\bar{\gamma} & =\sum_{i, j \geqslant 2} \bar{\gamma}_{i j}\left(y, x_{2}, \ldots, x_{n}\right) d x_{i} d x_{j}, \\
\gamma^{\prime} & =\sum_{i, j \geqslant 1} \gamma_{i j}^{\prime}\left(x, y, x_{2}, \ldots, x_{n}\right) d x_{i} d x_{j}, \quad \gamma_{i j}^{\prime}=O(x)=O(u v) .
\end{aligned}
$$

Utilisant $d y=\frac{1-u_{0}^{2}}{1+u_{0}^{2}} d v-\frac{4 u_{0} v}{\left(1+u_{0}^{2}\right)^{2}} d u$, et posant $\alpha_{0}=d u_{0}, \alpha_{1}=\frac{d v}{v}$ et $\alpha_{i}=\frac{d x_{i}}{v}$ pour $i>1$, nous obtenons le contrôle suivant :

$$
u_{0}^{2} g=d u_{0}^{2}+\frac{\left(1+u_{0}^{2}\right)^{2}}{4} \frac{d v^{2}+\bar{\gamma}}{v^{2}}+\sum_{i, j \geqslant 0} a_{i j} \alpha_{i} \alpha_{j},
$$

avec

$$
\begin{aligned}
& a_{00}=O\left(u_{0}^{3} v\right), \\
& a_{0 i}=O\left(u_{0}^{2} v\right), \quad i \geqslant 1, \\
& a_{i j}=O\left(u_{0} v\right), \quad i, j \geqslant 2 .
\end{aligned}
$$


Il en résulte immédiatement que le second membre de (31) est $O\left(u_{0} v\right)$, si bien qu'en réalité l'équation n'a pas de singularité en $v=0$ (dans $|d \phi|_{u_{0}^{2} g}^{2}$ apparaissent les dérivées $\partial_{u_{0}} \phi, v \partial_{v} \phi$ et $v \partial_{x_{i}} \phi$ qui ne sont pas singulières). Ainsi, l'existence d'une solution $\phi$ dans un voisinage de $\partial B$ ne pose pas de difficulté, et celle-ci s'annule en outre le long de $\{v=0\}$ : l'équation nous donne facilement un contrôle

$$
\phi=O\left(u_{0}^{2} v\right), \quad|d \phi|_{u_{0}^{2} g}=O\left(u_{0} v\right) .
$$

Posant $u=u_{0} e^{\phi}$, la métrique $g$ a donc la forme (29). En outre, dans un voisinage de $\partial B$, nous avons les coordonnées $\left(u=u_{0} e^{\phi}, v, x_{2}, \ldots, x_{n}\right)$; compte tenu de (35), (36) et (37), chaque métrique $\bar{g}_{u}$ est elle-même asymptotiquement hyperbolique sur $B$, avec infini conforme égal à $\bar{\gamma}$, donc indépendant de $u$.

Si deux telles métriques $g_{1}$ et $g_{2}$ coïncident à un ordre infini le long de $X$, il est clair aussi que les deux fonctions correspondantes $\phi_{1}$ et $\phi_{2}$, et les métriques $\bar{g}_{1}$ et $\bar{g}_{2}$, coïncident à un ordre infini le long de $\{x=0\}=\{u=0\} \cup\{v=0\}$. On peut alors faire les intégrations par parties menant à l'estimation de Carleman (16), et le reste de la démonstration est inchangée. Le théorème 3 est donc démontré.

\section{Démonstration du théorème 4}

Le cas d'un bord à distance finie se traite de la façon suivante. Soient $g$ et $g_{0}$ deux métriques lisses sur la variété à bord $M$. On identifie à nouveau un voisinage de $X$ dans $M$ au produit $\left[0, \epsilon\left[\times X\right.\right.$, avec coordonnées géodésiques, de sorte que $g=d x^{2}+\bar{g}$ et $g_{0}=d x^{2}+\bar{g}_{0}$. Le système des équations d'Einstein (5), (6) et (7), après avoir modifié la constante d'Einstein et remplacé $r$ par $x$, dit immédiatement que toutes les dérivées radiales de $\bar{g}$ et $\bar{g}_{0}$ coïncident en $x=0$. On applique alors la même méthode que pour le théorème 1 , en faisant les estimations par rapport à la métrique lisse $g_{0}$ : ainsi l'estimation (10) devient-elle, dès que $\lambda>-1 / 2$, pour une fonction $f$ s'annulant à un ordre infini en $x=0$,

$$
\int_{0}^{x_{0}}\left|\partial_{x} f\right|^{2} x^{-2 \lambda} d x \geqslant \lambda^{2} \int_{0}^{x_{0}}|f|^{2} x^{-2 \lambda-2} d x .
$$

Plus généralement, (12) devient

$$
\begin{aligned}
& \int_{0}^{x_{0}}\left|\nabla_{0}^{2}\left(\mathbb{I}-\mathbb{I}_{0}\right)\right|^{2} x^{-2 \lambda} d x \geqslant \\
& \quad C^{-1} \lambda^{2} \int_{0}^{x_{0}}\left(\left|\nabla_{0}^{2}\left(g-g_{0}\right)\right|^{2}+\lambda^{2} x^{-2}\left|\nabla_{0}\left(g-g_{0}\right)\right|^{2}+\lambda^{4} x^{-4}\left|g-g_{0}\right|^{2}\right) x^{-2 \lambda} d x
\end{aligned}
$$

et on a le même contrôle sur $H-H_{0}$ à la place de (14). Enfin, l'estimation de Carleman (16) devient, pour $\lambda \gg 0$ et $x_{0}$ assez petit,

$$
\begin{gathered}
\int_{0}^{x_{0}}|P s|^{2} x^{-2 \lambda} \mathrm{vol}^{g_{0}} \geqslant \\
C^{-1} \lambda^{-1} \int_{0}^{x_{0}}\left(\left|\nabla_{0}^{2} s\right|^{2}+\lambda^{2} x^{-2}\left|\nabla_{0} s\right|^{2}+\lambda^{4} x^{-4}|s|^{2}\right) x^{-2 \lambda} \mathrm{vol}^{g_{0}} .
\end{gathered}
$$

À partir de ces estimations, meilleures que dans le cas asymptotiquement hyperbolique, il devient clair que la démonstration du théorème 1 s'applique encore. 
Bien entendu, le même raisonnement s'applique au cas infinitésimal. En revanche, la méthode de localisation employée dans la section 4 dans le cas asymptotiquement hyperbolique ne peut plus être appliquée ici, puisque c'est toute la métrique, plutôt que sa classe conforme, qui est fixée au bord.

Remarque 5 (Unique continuation en un point). La même démonstration est valable pour deux métriques définies sur un ouvert au voisinage d'un point $p$, avec $x$ désignant la distance à $p$ : il suffit de raisonner en coordonnées normales. On obtient ainsi, sans recours aux coordonnées harmoniques, une démonstration alternative du fait que deux métriques d'Einstein, de même constante d'Einstein, égales au point $p$, dont les courbures et toutes leurs dérivées covariantes coïncident au point $p$, sont égales.

\section{Remerciements}

Luc Robbiano a répondu à mes questions sur la continuation unique à un stade préliminaire de ce travail. J'ai bénéficié de nombreuses discussions avec Marc Herzlich, qui m'a indiqué que M. Anderson et lui travaillent sur une approche différente de la continuation unique pour les métriques d'Einstein, maintenant parue dans [2]. Enfin, les commentaires de Rafe Mazzeo furent très utiles.

\section{Références}

[1] M. Anderson, Geometric aspects of the AdS/CFT correspondence, in O. Biquard, editor, AdS/CFT correspondence: Einstein metrics and their conformal boundaries, Vol. 8 of IRMA Lectures in Mathematics and Theoretical Physics, 1-31, European Mathematical Society (2005).

[2] M. T. Anderson and M. Herzlich, Unique continuation results for Ricci curvature and applications, J. Geom. Phys. 58 (2008), no. 2, 179-207.

[3] O. Biquard, Métriques d'Einstein asymptotiquement symétriques, Astérisque 265 (2000) vi+109. English translation: SMF/AMS Texts and Monographs 13 (2006).

[4] P. T. Chruściel, E. Delay, J. M. Lee, and D. N. Skinner, Boundary regularity of conformally compact Einstein metrics, J. Differential Geom. 69 (2005), no. 1, 111-136.

[5] C. Fefferman and C. R. Graham, Conformal invariants, Astérisque (1985), no. hors série, 95-116. The mathematical heritage of Élie Cartan (Lyon, 1984).

[6] C. R. Graham, Volume and area renormalizations for conformally compact Einstein metrics, in The Proceedings of the 19th Winter School "Geometry and Physics" (Srní, 1999), number 63 in Rend. Circ. Mat. Palermo (2) Suppl., 31-42 (2000).

[7] D. Helliwell, Boundary regularity for conformally compact Einstein metrics in even dimensions. arXiv:0705.2625 [math.DG].

[8] R. Mazzeo, Unique continuation at infinity and embedded eigenvalues for asymptotically hyperbolic manifolds, Amer. J. Math. 113 (1991) 25-45.

Université Pierre et Marie Curie-Paris 6, UMR 7586, Institut de Mathématiques de Jussieu, CASe 247, 4 Place Jussieu, 75252 PARIS CEDEX 05

E-mail address: biquard@math.jussieu.fr 